\title{
Células de la Cresta Neural y su Relación con Cardiopatía Congénita: Revisión Sistemática de la Literatura
}

\author{
Neural Crest Cells and their Relation to Congenital Heart Disease: \\ Systematic Review of the Literature
}

\author{
Víctor Ramos ${ }^{*, * *, * * * *}$ \& Ignacio Roa ${ }^{*, * * *, *, * * * * * * * *}$
}

RAMOS, V. \& ROA, I. Células de la cresta neural y su relación con cardiopatía congénita: revisión sistemática de la literatura. Int. J. Morphol., 34(2):489-494, 2016.

RESUMEN: Las cardiopatías congénitas corresponden al grupo de anomalías del desarrollo que se presentan con mayor frecuencia. Durante el desarrollo cardíaco participan distintos linajes celulares, donde destacan las Células de la Cresta Neural (CCN) por su amplia gama de derivados embriológicos y la susceptibilidad de afectar a múltiples sistemas si su función es alterada. El objetivo fue determinar el rol que cumplen las CCN durante el desarrollo cardíaco y las cardiopatías congénitas asociadas. Se diseñó un estudio descriptivo en base a una revisión sistemática de la literatura de las bases de datos MEDLINE y Scopus, utilizando la combinación de términos MeSH: ("Heart Diseases/congenital" OR "Heart Diseases/embriology" OR "Heart Diseases/etiology" OR "Heart Disesaes/ epidemiology") AND ("Neural Crest/abnormalities"). Se restringió la búsqueda a artículos de los últimos 10 años. De un total de 35 artículos obtenidos, 22 fueron incluidos para su revisión por estar relacionados con los objetivos de este estudio, excluyéndose duplicados entre bases de datos. Posteriormente se hizo un análisis individual y en conjunto de la información obtenida de los artículos seleccionados. La evidencia indica la participación directa o indirecta de las CCN durante la formación de las estructuras derivadas del polo arterioso del corazón en desarrollo, los grandes vasos arteriales y sus ramas colaterales, así como en su inervación y sistema de conducción. La alteración del funcionamiento normal de las CCN produce fenotipos cardíacos alterados, siendo la persistencia del tronco arterioso, doble salida ventricular derecha, defectos septales interventriculares y malformación de los aparatos valvares aórtico y pulmonar, los más frecuentes.

PALABRAS CLAVE: Cardiopatía congénita; Cresta neural; Células de la Cresta neural; Embriología.

\section{INTRODUCCIÓN}

La cardiopatía congénita corresponde a toda malformación cardíaca presente al momento del nacimiento, secundaria a alteraciones producidas durante la organogénesis del corazón (Neeb et al., 2013). Es sin duda, la anomalía de más altaincidencia en recién nacidos, con una tasa en Chile de entre 4 a 12 por cada 1000 nacidos vivos y una mortalidad correspondiente a $1 / 3$ del total de muertes causadas por anomalías congénitas y a 1/10 del total de todas las muertes en menores de un año (MINSAL, 2010), valores similares a los registrados en otras poblaciones a nivel mundial (van der Linde et al., 2011).

El desarrollo cardíaco es un proceso complejo donde participan y se coordinan diferentes poblaciones celulares; lo que explica, en parte, la alta prevalencia de anomalías congénitas asociadas a defectos cardíacos (Paul et al.,
2014). Las Células de la Cresta Neural (CCN) corresponden a una población celular multipotente originada de la porción dorsal del tubo neural, desde donde migran a través del organismo en desarrollo para dar origen a una amplia variedad de estructuras (Zhang et al., 2014) o participar en su formación, entre ellas el corazón, en donde más allá de la diferenciación en fenotipos celulares responsables de la formación de estructuras cardíacas, modulan factores de crecimiento y transcripción, coordinando así la acción de otros linajes celulares en el desarrollo del aparato cardiovascular (Brade et al., 2013).

Mediante modelos experimentales animales se ha logrado evidenciar que las alteraciones en el mecanismo normal de funcionamiento de las CCN produce fenotipos cardíacos alterados, en muchos casos asociados a malfor-

* Unidad de Morfología, Departamento de Ciencias Básicas Biomédicas, Facultad de Ciencias de la Salud, Universidad de Talca, Talca, Chile.

** Programa de Magister en Ciencias Biomédicas mención Patología Oral, Facultad de Ciencias de la Salud, Universidad de Talca, Talca, Chile.

**** Becario CONICYT-PCHA/Magíster Nacional/2016-22160548.

**** Programa de Doctorado en Ciencias Morfológicas, Facultad de Medicina, Universidad de La Frontera, Temuco, Chile.

******* Becario CONICYT-PCHA/Doctorado Nacional/2015-21150235. 
maciones de otros sistemas, reflejo de la variedad de funciones que desempeñan en el desarrollo embrionario (Nelms et al., 2011).

El objetivo de esta revisión sistemática de la literatura fue describir el rol que desempeñan las CCN durante el desarrollo cardíaco, así como establecer las principales cardiopatías derivadas de su alteración.

\section{MATERIAL Y MÉTODO}

Se diseñó un estudio descriptivo en base a una revisión sistemática de la literatura, aplicando una metodología de búsqueda (Fig. 1) en las bases de datos MEDLINE y Scopus, utilizando de manera combinada los siguientes términos MeSH: ("Heart Diseases/congenital" OR "Heart Diseases/embriology" OR "Heart Diseases/etiology" OR "Heart Diseases/epidemiology") AND ("Neural Crest/ abnormalities"). Se restringió la búsqueda a publicaciones realizadas entre el 01/01/2005 y la fecha de búsqueda (06/ 07/2015), para el desarrollo de una revisión actualizada a los último 10 años. No hubo restricción de idioma.

Se obtuvieron 11 artículos de la base de datos MEDLINE y 24 artículos de la base de datos Scopus. A partir de la lectura de resúmenes se seleccionaron 22 artículos relacionados con los objetivos del presente estudio, los que fueron incluidos para su revisión, excluyéndose artículos no relacionados y las coincidencias entre bases de datos.
Los artículos seleccionados fueron analizados a partir de su lectura y registro de datos de autor, año de publicación, tipo de estudio, tamaño muestral y principales conclusiones, si correspondía de acuerdo al tipo de artículo analizado.

\section{RESULTADOS}

De los 22 artículos seleccionados, 5 correspondieron a revisiones narrativas y 17 a estudios experimentales en animales, en los cuales fue utilizada recombinación genética con el fin de producir ratones knock-out en genes con conocida o posible relación sobre el desarrollo de las CCN, con la finalidad de observar el efecto de la intervención en el comportamiento de las $\mathrm{CCN}$ y el fenotipo presente en la descendencia tanto a nivel cardíaco como en otras estructuras asociadas, según reportaron algunos autores.

En la Tabla I se muestran los estudios experimentales revisados, donde se observa la amplia variedad de genes involucrados de manera conocida o supuesta, en el desarrollo y funcionamiento de las $\mathrm{CCN}$.

Los fenotipos cardíacos observados por los autores en sus investigaciones fueron variados, y su frecuencia dentro de los modelos experimentales aplicados está representada en la Figura 2. La principal anomalía congénita extra cardíaca asociada a la alteración de las CCN, observada por los autores, fue la presencia de malformaciones en el territorio craneofacial.

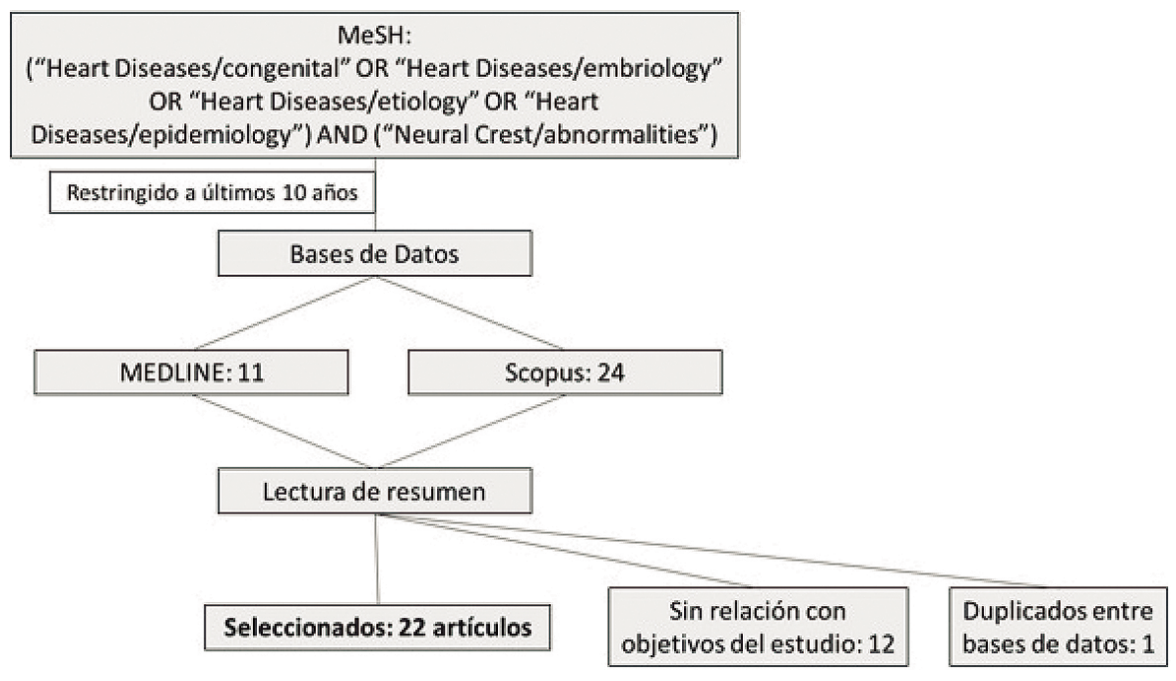

Fig. 1. Flujograma de la metodología de búsqueda para revisión sistemática. 
Tabla I. Metodología y principales resultados obtenidos de los estudios experimentales revisados.

\begin{tabular}{|c|c|c|c|c|}
\hline Autor (año) & $\mathbf{n}$ & Gen estudiado & Fenotipo cardíaco observado (*) & En asociación a \\
\hline Paul et al. (2014) & 238 & SoxC (Sox4 y Sox11) & PTA y DSVD & Malformación craneofacial \\
\hline Phillips et al. (2013) & 52 & Rock 1 y 2 & PTA y DSVD, DSIV y Displasia & --- \\
\hline Mommersteeg et al. (2013) & 28 & Robo 1 y 2 & Epicardio y polo venoso & --- \\
\hline Jain et al. (2011) & 22 & Pax 3 y Notch & VAB e DSIV & --- \\
\hline Nelms et al. (2010) & 166 & Foxd 3 y Pax 3 & PTA & $\begin{array}{c}\text { Malformación craneofacial y } \\
\text { del timo }\end{array}$ \\
\hline Huang et al. (2010) & 26 & Dicer & AAI, DSVD, DS IV & Malformación craneofacial \\
\hline Tang et al. (2010) & 22 & Smad-7 & $\begin{array}{c}\text { PTA, DSIV, hiperplasia valvar } \\
\text { arterial }\end{array}$ & Malformación craneofacial \\
\hline Zhang et al. (2010) & 46 & FGFR1 y FGFR2 & Hiperplasia valvar arterial & --- \\
\hline Nakamura et al. (2009) & --- & PTPN11 & $\begin{array}{l}\text { PTA, VAT y patrón arcos } \\
\text { arteriales anómalo }\end{array}$ & Malformación craneofacial \\
\hline Nie et al. (2008) & 93 & Smad-4 & $\begin{array}{l}\text { PTA, hipoplasia ventricular y } \\
\text { patrón arcos arteriales anómalo }\end{array}$ & Malformación craneofacial \\
\hline Porras \& Brown (2008) & 66 & Ablación $\mathrm{CCN}$ & $\begin{array}{l}\text { PTA, DSVD, DSIV, TGV, AAI, } \\
\text { patrón arcos arteriales anómalo }\end{array}$ & Malformación del timo \\
\hline Van de Putte et al. (2007) & 98 & Zfhx 1e & $\begin{array}{l}\text { Defecto del epicardio e hipoplasia } \\
\text { ventricular }\end{array}$ & $\begin{array}{c}\text { Malformación craneofacial, } \\
\text { gastrointestinal, SNA, SNP y } \\
\text { de melanocitos }\end{array}$ \\
\hline Liang et al. (2007) & --- & Pinch1 & $\begin{array}{l}\text { PTA, DSIV, defectos valvares y } \\
\text { patrón arcos arteriales anómalo }\end{array}$ & $\begin{array}{l}\text { Malformación craneofacial y } \\
\text { del timo }\end{array}$ \\
\hline Hutson et al. (2006) & 93 & FGF8 & PTA, TGV, EA/P & $\begin{array}{c}\text { Malformación craneofacial y } \\
\text { encefálica }\end{array}$ \\
\hline Park et al. (2006) & 206 & FGF8 & PTA, DSVD, TGV & --- \\
\hline Lepore et al. (2006) & 147 & GATA-6 & PTA, DSVD, DSIV, AAI & --- \\
\hline Oh et al. (2005) & 53 & MRTF-B & $\begin{array}{l}\text { PTA, ausencia de ducto arterioso, } \\
\text { vipoplasia ventricular }\end{array}$ & --- \\
\hline
\end{tabular}

* PTA= Persistencia del Tronco Arterioso; DSVD= Doble Salida Ventricular Derecha; DSIV= Defecto Septal Interventricular; VAB y VAT= Válvula Aórtica Bicúspide o Tetracúspide; CA= Coartación Aórtica; AAI= Arco Aórtico Interrumpido; TGV=Transposición de Grandes Vasos; EA/P= Estenosis Aórtica o Pulmonar.

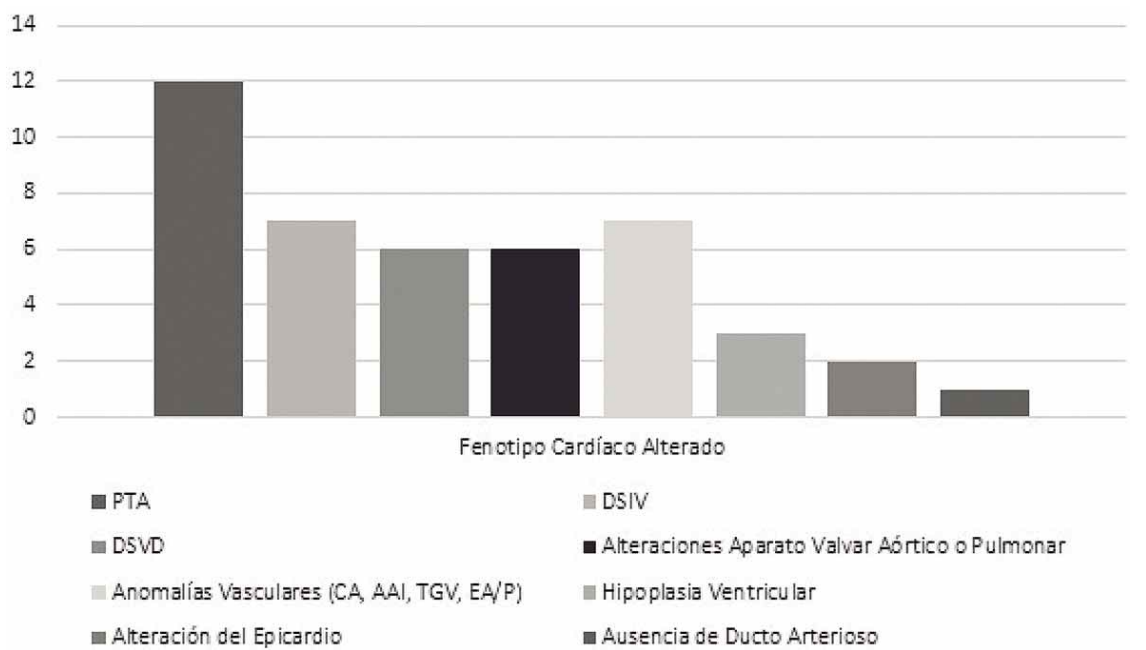

Fig. 2. Frecuencia de fenotipos cardíacos alterados en los estudios experimentales incluidos en la revisión.

\section{DISCUSIÓN}

El corazón es una de las primeras estructuras en formarse y adquirir capacidad funcional en el ser humano en desarrollo, y su constitución depende de la participación e interacción de diferentes grupos celulares.

A partir de la tercera semana de desarrollo intrauterino el mesodermo esplácnico de la región craneal del embrión se diferencia desarrollando una estructura tubular con forma de herradura denominada campo cardíaco primario, conformado por un revestimiento endotelial 
rodeado de cardioglía (gelatina cardíaca) y miocardio, a partir del cual se organiza la estructura general del corazón, diferenciandose, el polo venoso, atrios y parte del ventrículo derecho. El polo arterioso y la mayor parte de los ventrículos reciben aportes de células provenientes del mesodermo faríngeo, las que migran al corazón en desarrollo para conformar el campo cardíaco secundario, que ocupa la porción caudal y medial del campo cardíaco primario, diferenciándose en los cardiomiocitos que ocuparán los segmentos mencionados. Finalmente el proepicardio, un conglomerado de células migratorias derivadas del mesodermo esplácnico asociado al septo transverso, y adyacentes al polo venoso del corazón en formación, tiene como función formar el epicardio y los vasos coronarios (Okamoto et al., 2010; Brade et al.).

Las CCN originadas en la región comprendida entre la placoda ótica y el tercer par de somitos occipitales son denominadas $\mathrm{CCN}$ cardíacas, y migran desde el tubo neural hacia el corazón en desarrollo pasando por la región cervical, específicamente en relación al tercer, cuarto y sexto arco faríngeo, siendo el único linaje celular no mesodérmico que contribuye a la embriogénesis cardíaca. Su actividad en la formación del corazón es variada, contribuyendo a conformar la musculatura lisa del tracto efluyente (de salida) con su posterior división y parte del septo interventricular, además de modular la formación de los aparatos valvares aórtico y pulmonar, su inervación parasimpática y la organización de los patrones espaciales de los arcos aórticos y sus ramas colaterales (Sylva et al., 2014; Brade et al.).

El rol de las CCN en el desarrollo cardíaco fue establecido en la década de los 80 , a través de modelos experimentales en especímenes aviares, basados en la observación de embriones luego de la ablación de este linaje celular para el estudio de la inervación parasimpática del corazón. Accidentalmente se observó que el tracto efluyente de los especímenes estudiados no se dividía, dando inicio a una serie de estudios que buscaron identificar la acción de las $\mathrm{CCN}$ en el desarrollo del corazón, tanto desde el punto de vista estructural como molecular (Keyte \& Hudson, 2012).

La actividad de las CCN en la embriogénesis cardíaca es modulada por diferentes vías de señalización, a través de la expresión genes con un patrón temporal y espacial definido, ya sea en un estado pre migratorio en el tubo neural, durante su migración influenciada por agentes quimioatrayentes, o post migratorio al llegar al corazón en formación. En la última década diferentes modelos experimentales en ratones, a través de técnicas de recombinación génica, han permitido identificar algunos de los genes cuya expresión es relevante para la función normal de las CCN en el desarrollo cardíaco y como su supresión se traduce en fenotipos alterados, aunque las vías específicas de señalización no han sido dilucidadas por completo.

La evidencia obtenida de los modelos experimentales revisados indica que los fenotipos cardíacos más frecuentes asociados a defectos de CCN corresponden a la persistencia del tronco arterioso, doble salida ventricular derecha, defectos septales interventriculares y de los aparatos valvares aórtico y pulmonar, entre otros menos frecuentes. Por otro lado las cardiopatías congénitas más prevalentes a nivel mundial incluyen a los defectos septales interventriculares y Tetralogía de Fallot, que dentro de sus manifestaciones contempla comunicación interventricular, aorta cabalgante y doble salida ventricular derecha. mientras que dentro de las más graves, aunque menos frecuentes, se encuentra la persistencia del tronco arterioso, defectos de los aparatos valvares arteriales, transposición de grandes vasos y coartación aórtica (Marelli et al., 2007; van der Linde et al.), donde si bien estas dos últimas no se hayan dentro de los fenotipos observados con mayor frecuencia en modelos experimentales, su manifestación estaría ligada a alteraciones en el desarrollo de las CCN.

Estas observaciones sugieren que esta población celular es susceptible a sufrir alteraciones que conducen a anomalías del desarrollo, posiblemente debido a una mayor exposición en sus diferentes estadios de diferenciación y funcionamiento espaciotemporal.

Además del corazón, las CCN contribuyen a la formación del sistema nervioso periférico, meninges, melanocitos, odontoblastos, endocrinocitos medulares (células cromafines), y gran parte de los tejidos conectivos que forman el territorio cervical y craneofacial, incluyendo tejido óseo, cartilaginoso y muscular. Por ello no es extraño que una alteración de este linaje celular afecte a más de una estructura o sistema (Arteaga Martínez \& García Peláez, 2014).

Todos los autores que incluyeron en sus observaciones la alteración de otros territorios corporales evidenciaron anomalías craneofaciales, y con menor frecuencia anomalías a nivel del timo, encéfalo y sistema nervioso periférico, sugiriendo que estos territorios u órganos comparten vías de señalización común en torno a la expresión de genes específicos en CCN. Dicha situación explicaría los distintos niveles de compromiso que presentan algunos Síndromes que afectan al ser humano, como el Síndrome de deleción del cromosoma 22 (que incluye los Síndromes de Di Giorge, Velocardiofacial, y de Anomalía Facial Conotruncal), Síndrome de Treacher Collins y Síndrome de PHACE, entre otros, los que agrupan un conjunto heterogéneo de malfor- 
maciones que comprometen principalmente al territorio maxilofacial y cardíaco, con notables limitaciones funcionales que en muchos casos ponen en riesgo vital al individuo (Walker \& Trainor, 2006; Yutzey, 2010).

\section{CONCLUSIONES}

El rol cumplido por las CCN durante el desarrollo cardíaco ha sido comprobado mediante varios estudios de tipo experimental; los defectos asociados se traducen en alteraciones morfológicas y limitaciones funcionales, muchas veces letales, las cuales pueden presentarse de forma aislada o asociadas a síndromes, afectando así a múltiples sistemas, reflejo de la gran variedad de funciones que cumple esta población celular.

Conocer las bases del funcionamiento y participación de las $\mathrm{CCN}$ durante el desarrollo humano resulta fundamental al momento de pesquisar anomalías congénitas, ya sea durante los controles durante el período gestacional, o bien al momento del parto, donde la presencia de alteraciones craneofaciales u otros signos asociados pueden alertar al clínico ante la posibilidad de que el feto o neonato presente alguna cardiopatía congénita, tomando así las medidas perti-

RAMOS, V. \& ROA, I. Neural crest cells and their relation to congenital heart disease: Systematic review of the literature. Int. J. Morphol., 34(2):489-494, 2016.

SUMMARY: Congenital heart defects are the group of most frequent anomalies of development. Cardiac development in different cell lines, which include the Neural crest cells (NCC) for their wide range of embryological derivatives and susceptibility to affect multiple systems if their function is altered participate. The objective was to determine the role of the NCC during heart development and associated congenital heart disease. A descriptive study was designed based on a systematic review of the literature from the MEDLINE and Scopus data, using a combination of MeSH terms ("Heart Diseases / congenital" OR "Heart Diseases / Embryology" OR "Heart Diseases/etiology "OR" Heart Diseases/epidemiology ") AND ("Neural Crest/abnormalities"). Search for articles in the last 10 years was restricted. From a total of 35 articles retrieved, 22 were included related to the objectives of this study for review, excluding duplicated between databases. Subsequently, an individual and joint analysis was realized with the information from the selected items. Evidence indicates the direct or indirect involvement of NCC during the formation of the structures derived from arterial pole of the developing heart, the large arterial vessels and their collateral branches, as well as its innervation and conduction system. The disruption of normal operation of the NCC produces altered cardiac phenotypes, with the Persistence Truncus Arteriosus, Double-Outlet Right Ventricle, ventricular Septal Defects and malformation of the most common valvular aortic and pulmonary devices.

\section{KEY WORDS: Congenital heart disease; Neural crest; Neural crest cells; Embriology.}

nentes, con el fin de limitar el daño y mejorar su pronóstico.

\section{REFERENCIAS BIBLIOGRÁFICAS}

Arteaga Martínez, S. M. \& García Peláez, M. I. Embriología Humana y Biología del Desarrollo. México D. F., Médica Panamericana, 2014.

Brade, T.; Pane, L. S.; Moretti, A.; Chien, K. R. \& Laugwitz, K. L. Embryonic heart progenitors and cardiogenesis. Cold Spring Harb. Perspect. Med., 3(10):a013847, 2013.

Huang, Z. P.; Chen, J. F.; Regan, J. N.; Maguire, C. T.; Tang, R. H.; Dong, X. R.; Majesky, M. W. \& Wang, D. Z. Loss of microRNAs in neural crest leads to cardiovascular syndromes resembling human congenital heart defects. Arterioscler. Thromb. Vasc. Biol., 30(12):2575-86, 2010.

Hutson, M. R.; Zhang, P.; Stadt, H. A.; Sato, A. K.; Li, Y. X.; Burch, J.; Creazzo, T. L. \& Kirby, M. L. Cardiac arterial pole alignment is sensitive to FGF8 signaling in the pharynx. Dev. Biol., 295(2):486-97, 2006.

Jain, R.; Engleka, K. A.; Rentschler, S. L.; Manderfield, L. J.; Li, L.;
Yuan, L. \& Epstein, J. A. Cardiac neural crest orchestrates remodeling and functional maturation of mouse semilunar valves. J. Clin. Invest., 121(1):422-30, 2011.

Keyte, A. \& Hutson, M. R. The neural crest in cardiac congenital anomalies. Differentiation, 84(1):25-40, 2012.

Marelli, A. J.; Mackie, A. S.; Ionescu-Ittu, R.; Rahme, E. \& Pilote, L. Congenital heart disease in the general population: changing prevalence and age distribution. Circulation, 115(2):163-72, 2007.

Ministerio de Salud (MINSAL). Guía Clínica Cardiopatías Operables en Menores de 15 Años. Santiago de Chile, Ministerio de Salud (MINSAL), Gobierno de Chile, 2010. Disponible en: http:/ /www.supersalud.gob.cl/difusion/572/articles 571_guia_clinica.pdf

Mommersteeg, M. T.; Andrews, W. D.; Ypsilanti, A. R.; Zelina, P.; Yeh, M. L.; Norden, J.; Kispert, A.; Chédotal, A.; Christoffels, V. M. \& Parnavelas, J. G. Slit-roundabout signaling regulates the development of the cardiac systemic venous return and 
pericardium. Circ. Res., 112(3):465-75, 2013.

Nakamura, T.; Gulick, J.; Colbert, M. C. \& Robbins, J. Protein tyrosine phosphatase activity in the neural crest is essential for normal heart and skull development. Proc. Natl. Acad. Sci. U. S. A., 106(27):11270-5, 2009.

Neeb, Z.; Lajiness, J. D.; Bolanis, E. \& Conway, S. J. Cardiac outflow tract anomalies. Wiley Interdiscip. Rev. Dev. Biol., 2(4):499-530, 2013.

Nelms, B, L.; Pfaltzgraff, E. R. \& Labosky, P. A. Functional interaction between Foxd3 and Pax3 in cardiac neural crest development. Genesis, 49(1):10-23, 2011.

Nie, X.; Deng, C. X.; Wang, Q. \& Jiao, K. Disruption of Smad4 in neural crest cells leads to mid-gestation death with pharyngeal arch, craniofacial and cardiac defects. Dev. Biol., 316(2):417-30, 2008.

Lepore, J. J.; Mericko, P. A.; Cheng, L.; Lu, M. M.; Morrisey, E. E. \& Parmacek, M. S. GATA-6 regulates semaphorin 3C and is required in cardiac neural crest for cardiovascular morphogenesis. J. Clin. Invest., 116(4):929-39, 2006.

Liang, X.; Sun, Y.; Schneider, J.; Ding, J. H.; Cheng, H.; Ye, M.; Bhattacharya, S.; Rearden, A.; Evans, S. \& Chen, J. Pinch1 is required for normal development of cranial and cardiac neural crest-derived structures. Circ. Res., 100(4):527-35, 2007.

Oh, J.; Richardson, J. A. \& Olson, E. N. Requirement of myocardin-related transcription factor-B for remodeling of branchial arch arteries and smooth muscle differentiation. Proc. Natl. Acad. Sci. U. S. A., 102(42):15122-7, 2005.

Okamoto, N.; Akimoto, N.; Hidaka, N.; Shoji, S. \& Sumida, H. Formal genesis of the outflow tracts of the heart revisited: previous works in the light of recent observations. Congenit. Anom. (Kyoto), 50(3):141-58, 2010.

Park, E. J.; Ogden, L. A.; Talbot, A.; Evans, S.; Cai, C. L.; Black, B. L.; Frank, D. U. \& Moon, A. M. Required, tissue-specific roles for Fgf8 in outflow tract formation and remodeling. Development, 133(12):2419-33, 2006.

Paul, M. H.; Harvey, R. P.; Wegner, M. \& Sock, E. Cardiac outflow tract development relies on the complex function of Sox 4 and Sox11 in multiple cell types. Cell Mol. Life Sci., 71(15):2931-45, 2014.

Phillips, H. M.; Mahendran, P.; Singh, E.; Anderson, R. H.; Chaudhry, B. \& Henderson, D. J. Neural crest cells are required for correct positioning of the developing outflow cushions and pattern the arterial valve leaflets. Cardiovasc. Res., 99(3):452-60, 2013.

Porras, D. \& Brown, C. B. Temporal-spatial ablation of neural crest in the mouse results in cardiovascular defects. Dev. Dyn.,
237(1):153-62, 2008

Sylva, M.; van den Hoff, M. J. \& Moorman, A. F. M. Development of the human heart. Am. J. Med. Genet. A, 164A(6):1347-71, 2014.

Tang, S.; Snider, P.; Firulli, A. B. \& Conway, S. J. Trigenic neural crest-restricted Smad7 over-expression results in congenital craniofacial and cardiovascular defects. Dev. Biol., 344(1):233-47, 2010.

Van de Putte, T.; Francis, A.; Nelles, L.; van Grunsven, L. A. \& Huylebroeck, D. Neural crest-specific removal of Zfhx $1 b$ in mouse leads to a wide range of neurocristopathies reminiscent of Mowat-Wilson syndrome. Hum. Mol. Genet., 16(12):142336, 2007.

van der Linde, D.; Konings, E. E.; Slager, M. A.; Witsenburg, M.; Helbing, W. A.; Takkenberg, J. J. \& Roos-Hesselink, J. W. Birth prevalence of congenital heart disease worldwide: a systematic review and meta-analysis. J. Am. Coll. Cardiol., 58(21):2241-7, 2011.

Walker, M. B. \& Trainor, P. A. Craniofacial malformations: intrinsic vs extrinsic neural crest cell defects in Treacher Collins and 22q11 deletion syndromes. Clin. Genet., 69(6):471-9, 2006.

Yutzey, K. E. DiGeorge syndrome, Tbx1, and retinoic acid signaling come full circle. Circ. Res., 106(4):630-2, 2010.

Zhang, D.; Ighaniyan, S.; Stathopoulos, L.; Rollo, B.; Landman, K.; Hutson, J. \& Newgreen, D. The neural crest: a versatile organ system. Birth Defects Res. C Embryo Today, 102(3):275-98, 2014.

Zhang, J.; Chang, J. Y.; Huang, Y.; Lin, X.; Luo, Y.; Schwartz, R. J.; Martin, J. F. \& Wang, F. The FGF-BMP signaling axis regulates outflow tract valve primordium formation by promoting cushion neural crest cell differentiation. Circ. Res., 107(10):1209-19, 2010.

Dirección para correspondencia:

Víctor Ramos Vega

Departamento de Ciencias Básicas Biomédicas

Facultad de Ciencias de la Salud

Universidad de Talca

Av. Lircay s/n, Talca

CHILE

Email: vramos@utalca.cl

Recibido: 10-02-2016

Aceptado:16-04-2016 